\title{
MODELING, TOPOLOGY OPTIMIZATION AND EXPERIMENTAL VALIDATION OF GLASS-TRANSITION-BASED 4D-PRINTED POLYMERIC STRUCTURES
}

\author{
SARA A. PAKVIS*, GIULIA SCALET ${ }^{\dagger}$ STEFANIA MARCONI ${ }^{\dagger}$ \\ FERDINANDO AURICCHIO ${ }^{\dagger}$ AND MATTHIJS LANGELAAR* \\ *Delft University of Technology \\ Mekelweg 2, 2628 CD Delft, The Netherlands \\ e-mail: sarapakvis@hotmail.com,m.langelaar@tudelft.nl, web page: \\ https://www.tudelft.nl/ \\ ${ }^{\dagger}$ University of Pavia \\ Corso Str. Nuova, 65, 27100 Pavia, Italy \\ e-mail: giulia.scalet@unipv.it, stefania.marconi@unipv.it, \\ ferdinando.auricchio@unipv.it, web page: http://wcm-3.unipv.it/
}

Key words: 4D-Printing, Glass Transition, Shape Memory Polymer, Topology Optimization

\begin{abstract}
In recent developments in the field of multi-material additive manufacturing, differences in material properties are exploited to create printed shape memory structures, which are referred to as $4 \mathrm{D}$-printed structures. New printing techniques allow for deliberate introduction of prestresses in the specimen during manufacturing. This prestress is combined with a heat-induced glass transition, which lowers the materials Young's modulus. Upon the decrease in stiffness, the prestress is released, which results in the realization of a pre-programmed deformation. Coupled with the right design, this enables new functionalities. As the design of such functional multi-material structures is crucial but far from trivial, a systematic methodology is developed, where a finite element model is combined with a density-based topology optimization method to describe the material layout. The coupling between the definition of the prestress and the material interpolation function used in the topology description is addressed. The efficacy of topology optimization to design 4D-printed structures is explored by applying the methodology to a variety of design problems. Tests are performed with printed samples to calibrate the prestress and to validate the modeling approach. This study demonstrates that by combining topology optimization and 4D-printing concepts, stimuli-responsive structures with specific properties can be designed and realized.
\end{abstract}


Sara A. Pakvis, Giulia Scalet, Stefania Marconi, Ferdinando Auricchio and Matthijs Langelaar

\section{Introduction}

Additive manufacturing or 3D-printing is a manufacturing technique where a material is deposited layer-wise to create three-dimensional structures. This technology can easily deal with extremely complex geometries, which would be very difficult or even impossible to create using traditional manufacturing techniques. Since its invention in the 1980s, 3D-printing has evolved into a versatile and widely used method. It is used in application fields such as bio-mechanics, prototyping, and construction ${ }^{1}$. With the development of new methods, materials, and equipment over the past few decades, new possible applications arise. One of those newer applications is to use 3D-printing to create smart, responsive structures. This type of 3D-printing is referred to as ' $4 \mathrm{D}$-printing'", where time is referred to as the fourth dimension. The initial structures evolve into different configurations over time under the influence of a stimulus, such as heat, water or pH. Such a technique has interesting potential application fields: using 4D-printing, self(dis)assembling, smart-behaving, and self-repairing structures can be created $^{3}$.

By carefully engineering the topology of the initial structure, a desired deformed shape can be obtained after 4D-transformation. However, to intuitively design a structure such that it deforms in the desired way is not trivial. Combining 4Dprinting with automated design approaches such as topology optimization seems

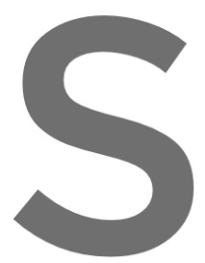
promising. Topolog
find the optimal ma
best structural perf
printed structurds.
variant of topoldor gy optimization is a m
materia placement in a
The so-called densit
Theptimization 4 in
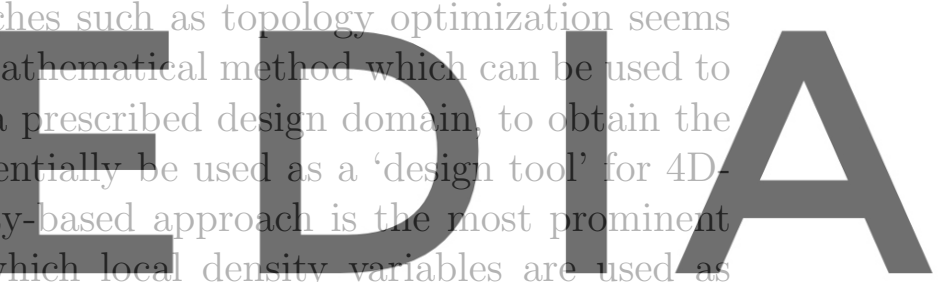

design variables to describe the material layout. This approach will also be used

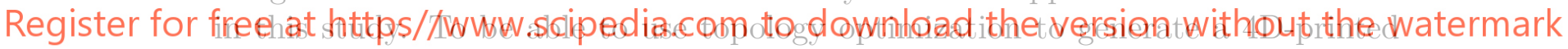
structure, it should be modelled numerically, which is a challenge in itself.

Some progress has already been made in the field of modeling $4 \mathrm{D}$-printed structures in combination with topology optimization, and even a few experiments have already been reported. In the work of Maute et al. ${ }^{5}$, numerically modeled structures are optimized with a level-set topology optimization method to match simple target shapes such as a parabola and a sinusoid. These optimized structures were printed and experimentally tested. Geiss et al. ${ }^{6}$ use a combination of a level-set and a density-based topology optimization method to create a three-dimensional model of an active propeller. A three-dimensional adaptive wing design with three different materials is generated using a genetic algorithm-based optimizer in the study of Sossou et al. ${ }^{7}$. In a later study by Geiss et al. ${ }^{8}$, more complex transformed shapes are achieved, such as a figure-eight and a gripper. These obtained designs were also printed and tested, and the numerical results matched the experimental results very well. In the current state-of-the-art, material models that are used to 
a

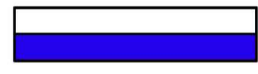

b

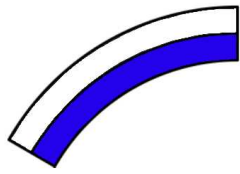

Figure 1: Bending behaviour of a bi-layer sample with active material (white) and passive material (blue), a) before transformation, b) after transformation.

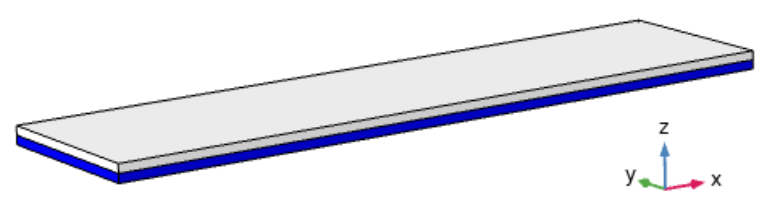

Figure 2: Three-dimensional geometry with active material (white) and passive material (blue).

model 4D-printing polymer structures are often rather complex, and also the levelset and genetic algorithm-based approaches used in previous studies are not trivial to implement in commercial software. In order to be able to practically combine 4D-printing with topology optimization, the need for a simpler material model arises to reduce computation time and to allow for sensitivity analysis. To further reduce complexity, a density-based topology optimization approach for generating $4 \mathrm{D}$ printed structures is developed.

\section{Amorphous Polymer 4D-Printing}

This research is focused on a specific type of 4D-printing with amorphous polymers, where the working principle relies on glass transition. One of the polymers is referred to as the passive material. This material undergoes a glass transition
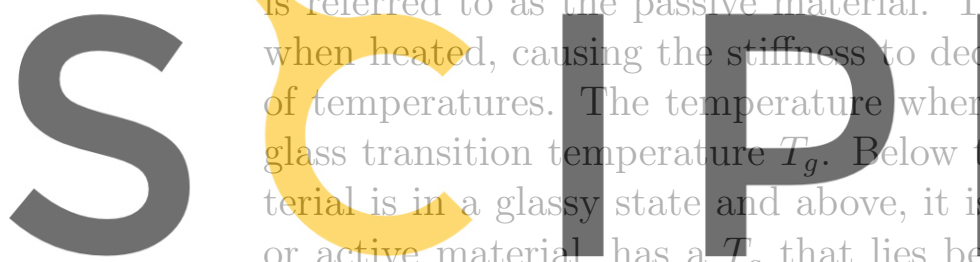

or active materiction has
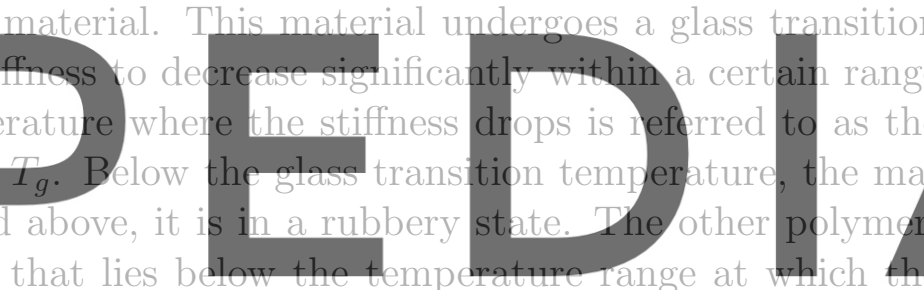

structure is heated. Therefore, this material is in the rubbery state at all times

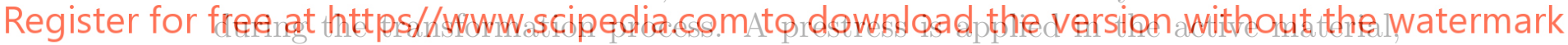

on which the passive material is printed to create a bi-layered structure. When the

glassy polymer is heated above its glass transition temperature, it allows for the rubbery polymer to release the prestress. This causes the structure to deform from its initial configuration (Fig. 1a) towards the transformed configuration (Fig. 1b). Using the glass transition mechanism of amorphous polymers is a popular choice in structural applications such as self-(dis)assembly ${ }^{9-12}$. The polymers have a relatively high stiffness compared to other $4 \mathrm{D}$-printing materials. Another advantage is that the transformed configuration of the structure is stable: the structure does not transform back to the original configuration when the stimulus is removed. The recent development of a direct $4 \mathrm{D}$-printing process ${ }^{13}$ allows for omitting all the steps of programming the prestress into the active material, which makes the fabrication process considerably faster. It can be concluded that this type of 4Dprinting is well suited for creating relatively stiff, fast, and stable structures. 
Sara A. Pakvis, Giulia Scalet, Stefania Marconi, Ferdinando Auricchio and Matthijs Langelaar

Table 1: Dimensions of the Modeled Geometry

\begin{tabular}{|c|c|c|}
\hline Parameter & Symbol & Value $(\mathbf{m m})$ \\
\hline Height of passive layer & $h_{p}$ & 0.5 \\
\hline Height of active layer & $h_{a}$ & 0.5 \\
\hline Length & $L$ & 40 \\
\hline Width & $w$ & 10 \\
\hline
\end{tabular}

Table 2: Material Properties of TPU and ABS

\begin{tabular}{|c|c|c|}
\hline Property & TPU & ABS \\
\hline Young's modulus & $12 \mathrm{MPa}$ & see Fig. 3 \\
\hline Poisson's ratio & 0.48 & 0.3 \\
\hline Mass density & $1.47 \mathrm{~g} / \mathrm{cm}^{3}$ & $1.11 \mathrm{~g} / \mathrm{cm}^{3}$ \\
\hline$T_{g}$ & $-35^{\circ} \mathrm{C}$ & $85^{\circ} \mathrm{C}$ \\
\hline
\end{tabular}

\section{Modeling Approach}

\subsection{Model Definition}

To simulate the transformation process of printed polymer structures, a numerical model is created in 2D and 3D. Although the design freedom is limited in 2D, it has the benefit that the simulation is less computationally intensive because of the lower number of degrees of freedom. If only in-plane bending occurs, a 2D model can accurately represent the transformation behaviour. But when for example outof-plane bending or twisting come into play, a 3D model is necessary. A bi-layer structure is modeled with the dimensions stated in Table 1 , to obtain the geometry shown in Fig. 2. All boundaries are unconstrained, except for the rightmost edge. In 2D, this edge has a roller boundary condition combined with a fixed point.
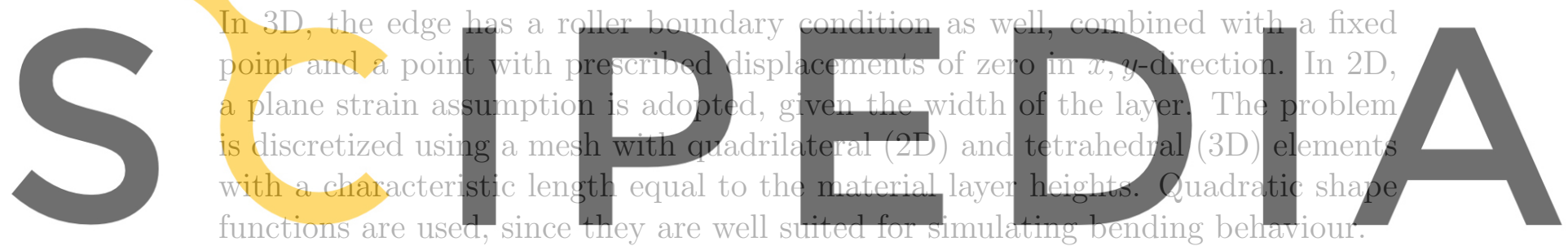

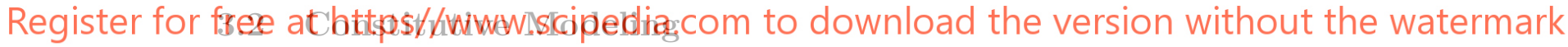

Two different materials are going to be used during printing. Thermoplastic polyurethane (TPU) is used for the active parts and acrylonitrile butadiene styrene (ABS) is used for the passive parts of the structure. The relevant material properties of these materials can be found in Table 2. The Young's modulus $E$ of ABS is highly dependent on the temperature $T$. To accurately describe the this relation within a certain temperature range, the data are fitted to the following equation $^{14}$ :

$$
E(T)=\left(E_{r}-E_{g}\right) \exp \left(-\left(\frac{T}{T_{1}}\right)^{m_{1}}\right)+E_{g} \exp \left(-\left(\frac{T}{T_{2}}\right)^{m_{2}}\right)
$$

where $E_{r}$ and $E_{g}$ represent the Young's modulus at the beginning of the rubbery and glassy plateau. $T_{1}$ and $T_{2}$ are the temperatures at the beginning of the glass transition and melting transition, respectively. Weibull moduli $m_{i}$ correspond to the statistics of bond breakage. Published data ${ }^{15}$ resulting from sample tests are used to find the relation between $E$ and $T$. The fitted curve is shown in Fig. 3. 


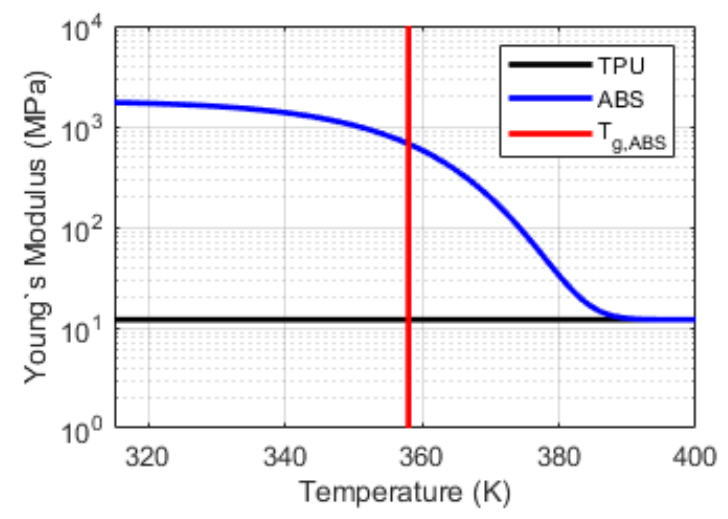

Figure 3: $E-T$ curve of TPU and ABS

Since large displacements occur, geometrical nonlinearities are considered in the model. The strain is represented by the Green-Lagrange strain tensor and the stresses are represented by the Second Piola-Kirchhoff tensor. The material behaviour of elastomers is often described by a hyperelastic material model. However, for the considered bilayer structure, simulation results with a hyperelastic material model and a linear elastic model hardly show any difference. Therefore,

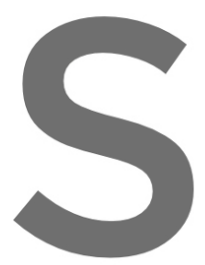
nonlinearities in the stress-strain relation
tive relation can simply be described by
to be isotropic. Time-dependent effects
by fast changes in the loading porramet
assumed that indrtial forces can be ignd
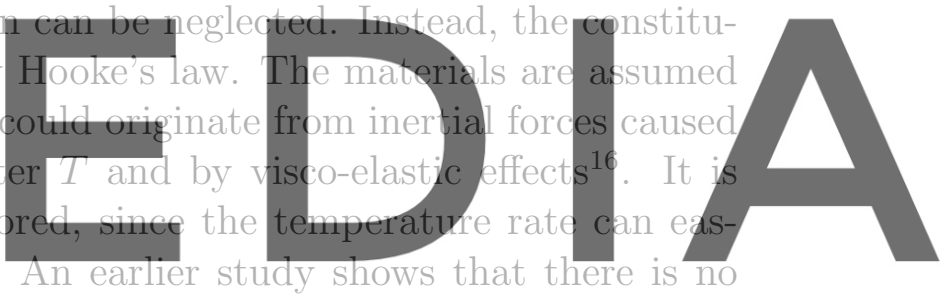
ily be controlled to be sufficiently low. An earlier study shows that there is no Register for free at https./www scipedia com to download the version without the watermark

\section{Density-Based Topology Optimization}

This section describes relevant aspects of density-based topology optimization of $4 \mathrm{D}$-printed structures. The optimal layout of a structure is generated within a specified domain, using a density field to describe the material distribution in the domain, and a penalization to steer the density towards 0 (void) or 1 (solid material $)^{4}$. Intermediate density values are unwanted and can be difficult to realize in practice. These intermediate values are often referred to as grey areas. The optimization problem consists of an objective function that should either be maximized or minimized and which is possibly subjected to constraints. 


\subsection{Penalized Variables}

The Young's modulus of the material layer that is optimized, is penalized such that less stiffness is provided for intermediate density values. The penalized Young's modulus $E\left(\theta_{p}\right)$ is obtained by multiplying the Young's modulus $E_{0}$ of the optimized material with the penalized material factor $\theta_{p}$ :

$$
E\left(\theta_{p}\right)=E_{0} \theta_{p}
$$

In the Simplified Isotropic Material with Penalization (SIMP) method, the penalized material volume $\theta_{p}$ is given by $^{17}$ :

$$
\theta_{p}=\theta_{\min }+\left(1-\theta_{\min }\right) \theta^{p}
$$

where $p \geq 1$ the penalization factor, which makes intermediate density values provide less stiffness relative to their weight. Increasing $p$ leads to a decrease of grey areas in the topology. Usually, the penalization factor is chosen as $p=3$. The $\theta_{\text {min }}$ term (where $\theta_{\min }>0$ ) is introduced to make sure the penalized Young's modulus $E\left(\theta_{p}\right)$ is never equal to zero, which is done for numerical reasons. The printing stress is modeled as an initial stress in the top layer material. To avoid stress being assigned to intermediate density values, it is essential to penalize the
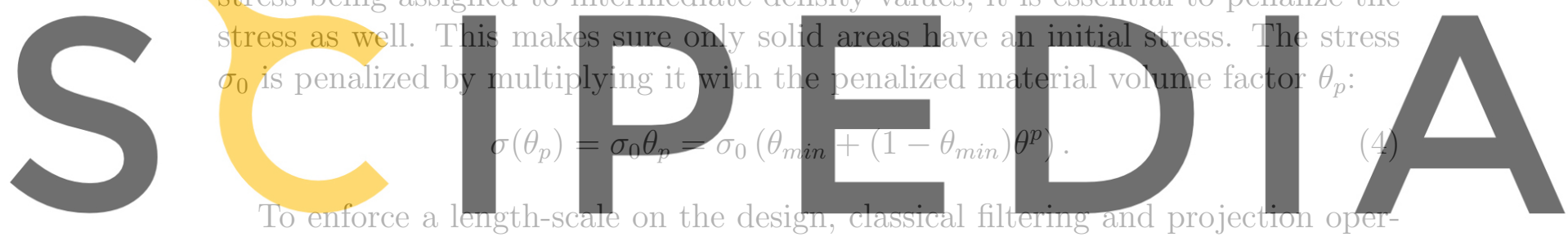

ations are included ${ }^{18,19}$.

free at ho

\subsection{Objective Function}

The target shape is approached by matching a desired displacement to the actual displacement. The goal of the optimization is to minimize the difference between nodal displacement and target displacement. The following objective function is used:

$$
f=w\left(\frac{v-v_{\text {target }}}{v_{\text {target }}}\right)^{2}+(1-w) V,
$$

where $v$ is the vertical tip displacement and $v_{\text {target }}$ is the targeted vertical tip displacement. The first term, $\left(\frac{v-v_{\text {target }}}{v_{\text {target }}}\right)^{2}$, is what can be referred to as a 'displacement matching' function, because the difference between the displacement and the targeted displacement is minimized. This term can be slightly modified to include multiple target points on the structure or to match the displacement of an entire edge to a target function. The second term, $V$, is the average material volume fraction. Minimizing this normalized volume further helps in decreasing 
grey areas in the optimization. The weight factor $0 \leq w \leq 1$ is introduced to be able to change the importance of the different terms in the function. Numerical experiments show that a weight factor of $w=0.9$ leads to both a well-defined topology and a sufficient displacement match.

\section{Numerical Examples}

\subsection{Parabola (2D)}

The modeling approach and topology optimization method can be used to match a transformed 4D-printed object to a target function of the form:

$$
f(x)=-A\left(\frac{2(x-L)}{L}\right)^{n},
$$

which represents a curve in the $x, y$-plane. The initial geometry is a simple bi-layered structure with dimensions as reported in Table 1. Starting from this geometry, the density-based topology optimization method is applied to the active material domain. In Fig. 4a can be seen that a good match with the target curve is obtained, through a material layout consisting of adequately placed disconnected patches of the active material.
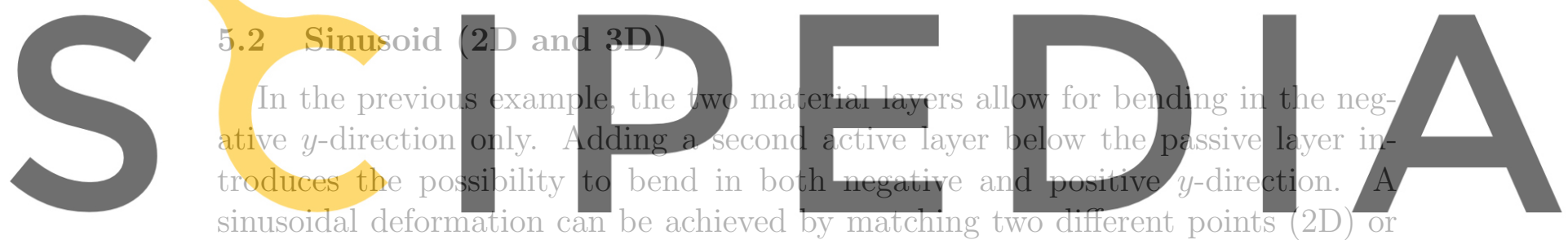
Register forges (3D) on the surface of the structure to a targeted displacement. The first free at https/ www scipedia.com to download the versign without the watermark the beam. The results can be found in Fig. 4b and Fig. 4c. In both the 2D and 3D case, the actual displacements approach the targeted displacements well. The slight difference in material distribution between the two cases can be explained by the fact that the optimizer has an additional design freedom in the in-plane direction.

\subsection{Twisting deformation (3D)}

Doing simulations in 3D allows for assigning two different target displacements in what would be the out-of-plane direction in 2D. By doing this, a twisting deformation can be obtained. A topology optimization is performed with an initially flat, square bilayer structure. The two tip corners are assigned target displacements. The resulting design in Fig. 5 shows a distinctive pattern of diagonal stripes, which are created by the optimizer to achieve the specified combination of both twisting and bending deformation. 


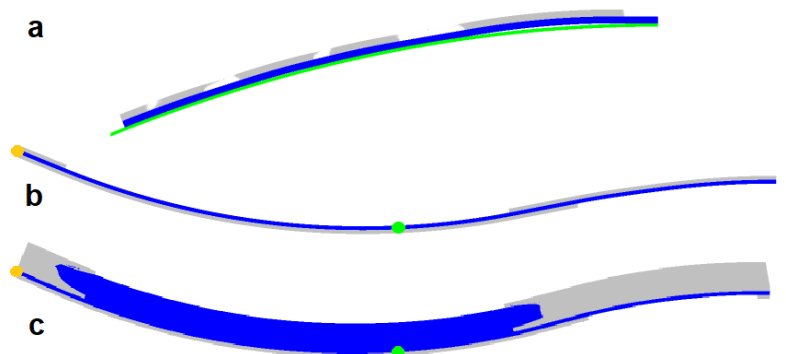

Figure 4: Optimized geometries with active material (grey) and passive material (blue) in transformed configuration, a) parabola (target shape where $A=0.002 \mathrm{~m}$ and $n=2$ indicated in green), b) sinusoid (2D), c) sinusoid (3D). Probe point 1 (tip) is indicated in orange and probe point 2 (middle) is indicated in green, $v_{1, \text { target }}=2 \mathrm{~mm}$ and $v_{2, \text { target }}=-3 \mathrm{~mm}$.

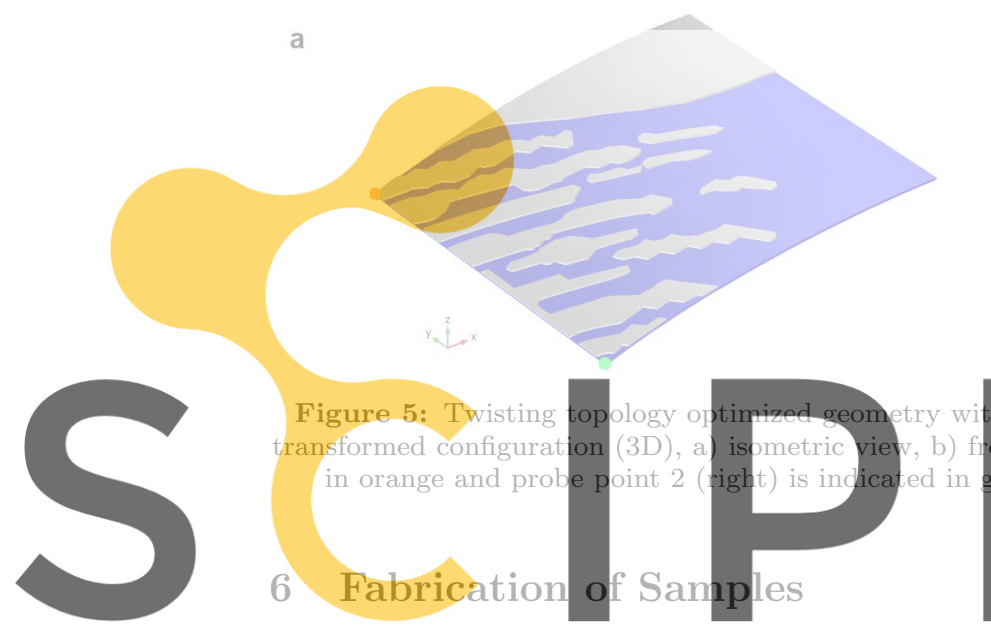

b

The 3D-printing technology employed is Fused Filament Fabrication (FFF).

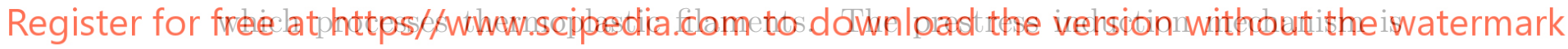
based on the temperature gradient. The employed polymers tend to expand when heated, while the cooling causes their shrinkage. The two parameters mainly acting on the prestress induction are printing velocities and temperatures. In addition to the two printing materials discussed in Section 3.2, High Impact PolyStyrene (HIPS) is used as support material to create the base on which the active material is deployed. HIPS is selected specifically for its chemical compatibility with TPU, which allows the two materials to stick together during the printing and to easily detach once the temperature has lowered.

The 3D-printing of the samples is carried out by means of a 3NTR A4v3 FFF printer, endowed with three extruders and a heated plate. Three copies of each configuration are pre-arranged and locked on the chosen coordinates. A dedicated printing profile is created in the slicer, where the main issue is to guarantee a good adhesion between ABS and TPU, for which printing temperatures and velocities have the highest impact on the outcome. The following parameters are used: i) 
layer thickness of $0.24 \mathrm{~mm}$, to ensure the highest dimensional accuracy according to the printer resolution; ii) extrusion width of $0.4 \mathrm{~mm}$, iii) rectilinear aligned infill, with extrusion lines parallel to the longest side of the beam in all the layers; iv) one perimeter. The ABS, TPU and HIPS are printed at $240^{\circ} \mathrm{C}, 230^{\circ} \mathrm{C}$ and $250^{\circ} \mathrm{C}$ respectively. The printing bed temperature is set to $110^{\circ} \mathrm{C}$. The printing velocity is set to $20 \mathrm{~mm} / \mathrm{s}$ for TPU and ABS and to $40 \mathrm{~mm} / \mathrm{s}$ for HIPS.

\section{Experiments}

As described earlier, the 4D-transformation of samples relies on the prestress in the active material. However at the present state, not enough data is available to precisely tune the stress introduced by the printing process, given the number of parameters which influence the outcome. For that reason, the effect of different thicknesses $h_{p}$ and $h_{a}$ is focused on. The geometries of the sample groups are shown in Fig. 6 and Table 3. The data obtained during these experiments represent the relation between material layer height and deformation, and are used to calibrate the numerical model to find the prestress. Once this prestress is found, the topology optimized structures shown in Fig. $4 \mathrm{c}$ and 5 can be generated, printed and tested. These designs are referred to as sample F and G, and they are based on

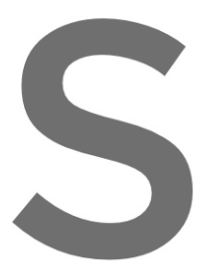
the optimizations described earlier.
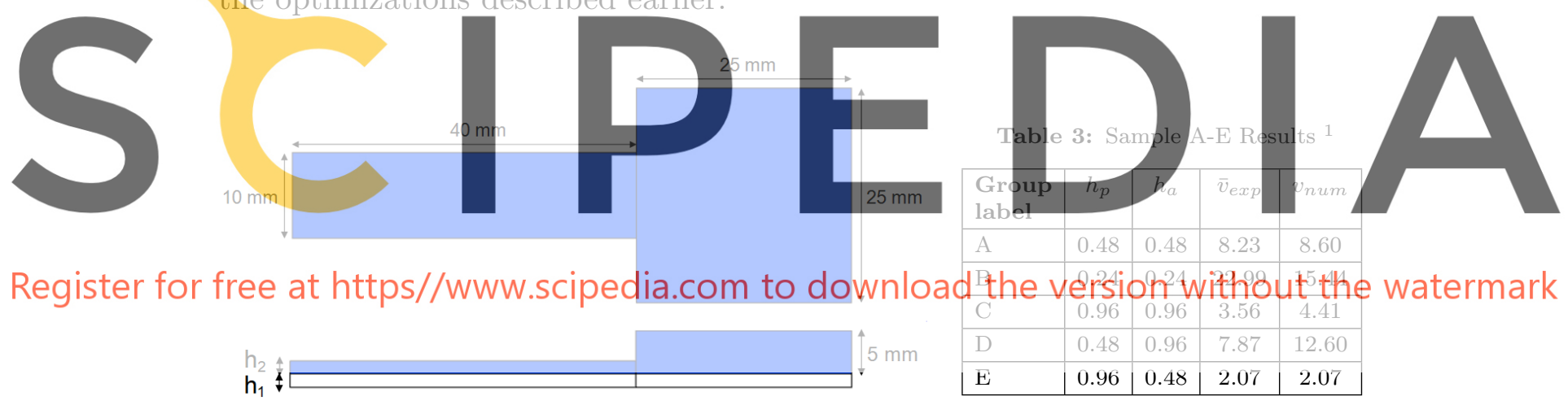

Figure 6: Prestress calibration sample dimensions, TPU (white) and ABS (blue)

The printed samples are heated above the $T_{g}$ of the ABS through immersion in a water bath at $98{ }^{\circ} \mathrm{C}$ (Fig. 7a). The temperature of the water is controlled using a heating immersion circulator, and an ad-hoc gripping system (Fig. 7b) is adopted to ensure the fixing of the square enlargement. The samples were kept immersed for 5 minutes to ensure the completion of the transformation process. After immersion, the deflection of each of these beam-shaped samples is measured. Three samples are tested for sample groups A-E, while one sample was tested for samples $\mathrm{F}$ and $\mathrm{G}$.

\footnotetext{
${ }^{1}$ All values in this table are given in $\mathrm{mm}$.
} 


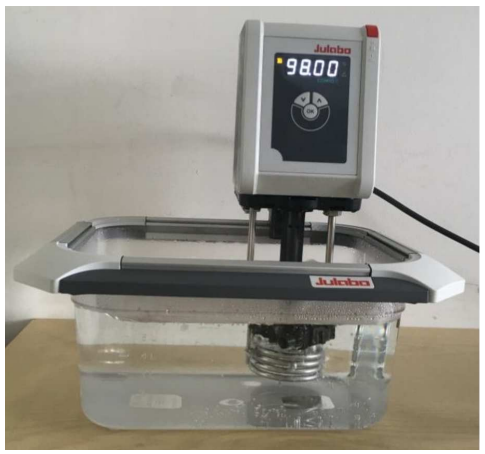

(a) Test setup

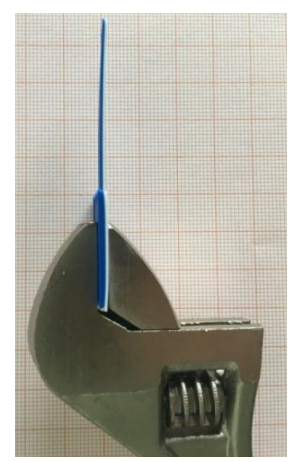

(b) Clamp

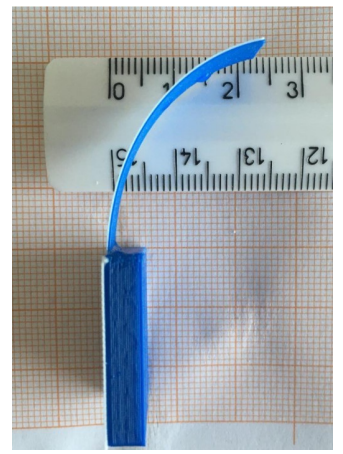

(c) Bent sample

Figure 7: Experimental setup for prestress calibration

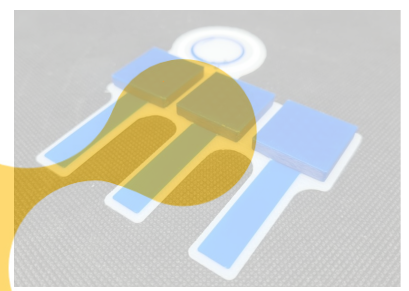

(a) Samples A-E

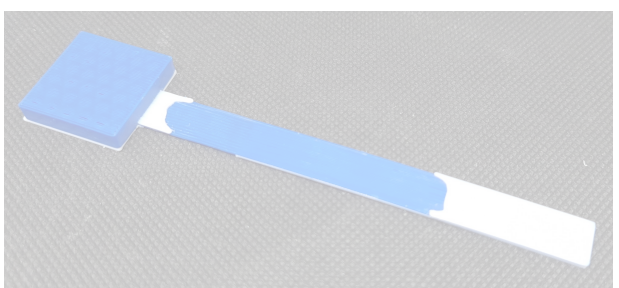

(b) Sample F

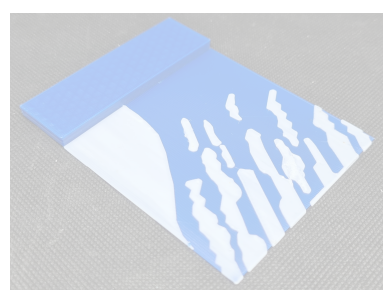

(c) Sample G
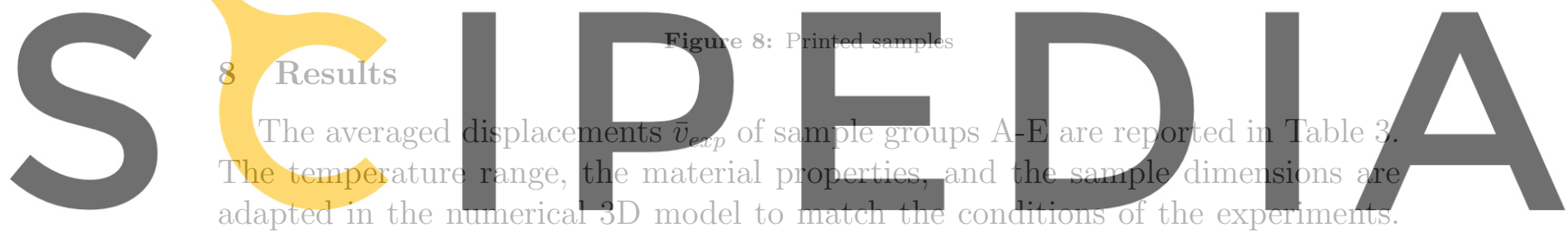

Numerical simulations are run for different prestress values, and subsequently the

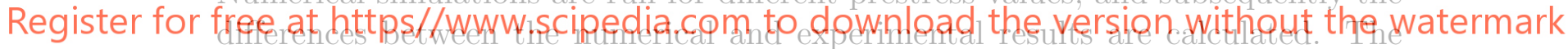

prestress for which this average deviation is minimal, is chosen as the calibrated prestress. From the numerical simulations can be concluded that this is the case for $\sigma_{0}=270 \mathrm{kPa}$, where the deviation is $2.70 \mathrm{~mm}$. A comparison of the experimental and numerical results for the calibrated prestress can be found in Fig. 9. For sample groups A, C and D, there is a good agreement between the results. However for sample group B, the displacement is underestimated by the simulation, and for sample group D, the displacement is overestimated. Although the exact reason of the unexpected results is unknown, there are several possible explanations: i) the prestress might not be the same in all sample groups, because the thickness of the material domains might have an influence on the thermal expansion process that introduces the prestress, ii) a thin sample may not have enough layers to assume an isotropic material model, iii) the diverging result can be caused by assumptions made in the modeling approach, such as the linear elastic material model.

Printed samples $\mathrm{F}$ and $\mathrm{G}$ are shown in Fig. 8. Test results of these samples 


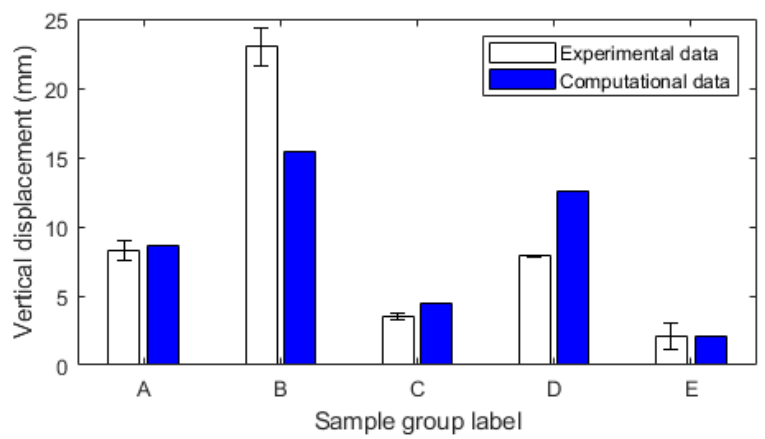

Figure 9: Comparison of experimental and numerical results of beam-shaped samples

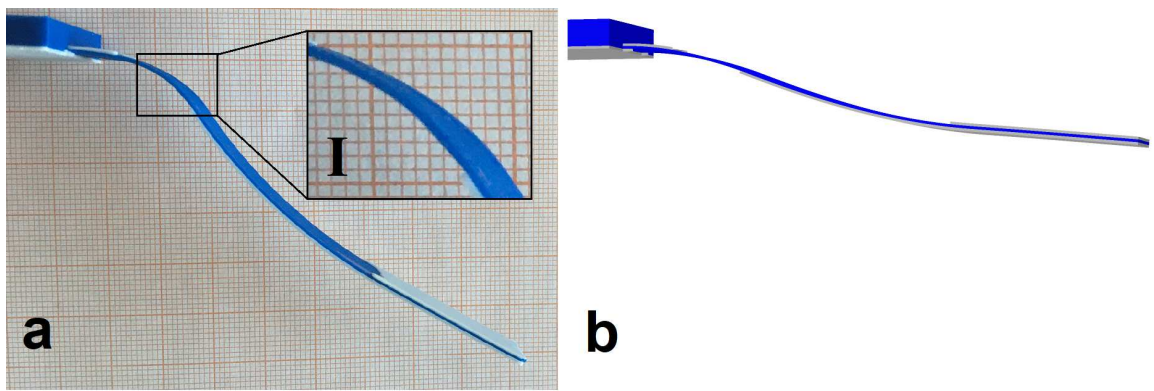

Figure 10: Sample F deformation, a) experimental, b) numerical (gravitational effects included).

can be found in Table 5. For sample F, probe point 1 is located in the middle of the beam and probe point 2 is located at the tip. In this sample, bending occurs where the sample has only a single passive layer (indicated as section I in Fig. 10). Because of this unexpected behaviour, the experimental displacements are much larger than predicted by the numerical model. With gravitational effects included the approximation is improved, but the displacements are still underestimated in the simulation. Apart from the single material section (section I), the experimental and numerical deformations are similar. The deformation in sample $\mathrm{G}$ is shown in Fig. $4 \mathrm{~b}$ and c, where probe point 1 is the left free corner and probe point 2 is the right free corner. The difference in displacement between these two points indicates the amount of twist, which is larger in the printed sample. The difference in amount of twist could be explained by the fact that this sample is very irregular, which could affect the prestress in the active material.

\section{Conclusion}

The use of topology optimization to design 4D-printed structures has been explored by using the density approach combined with a numerical modeling approach. The capabilities of the modeling and topology optimization approach are shown by several variations of the original. It can be expanded such that it 


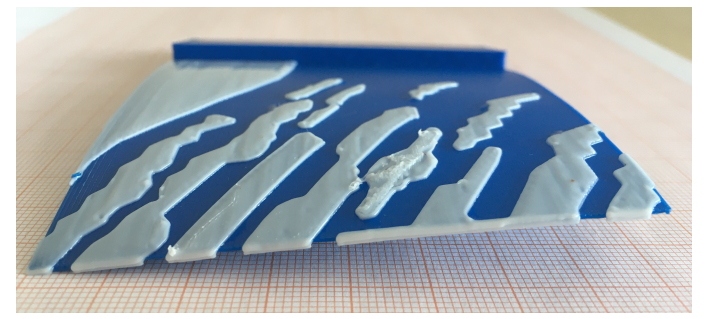

Table 4: Sample G deformation.

Table 5: Topology Optimization Sample Results

\begin{tabular}{|l|c|c|c|c|}
\hline $\begin{array}{l}\text { Group } \\
\text { label }\end{array}$ & $\bar{v}_{1, \exp }$ & $\bar{v}_{2, \text { exp }}$ & $v_{1, \text { num }}$ & $v_{2, \text { num }}$ \\
\hline $\mathrm{F}$ & -30 & -53 & -12.6 & -18.6 \\
\hline $\mathrm{G}$ & -15 & -26 & -15.8 & -19.0 \\
\hline
\end{tabular}

matches the displacements of multiple points in the material domain to multiple target displacements. Another variation is to match an entire surface to a target function. Besides bending transformation, a twisting transformation can be obtained by changing the target displacement settings in the optimization. The number of layers can be expanded, such that the range of target displacements becomes larger and more complex target shapes can be obtained.

The modeling and topology optimization approach was validated by experimental results. The initial stress introduced by the direct $4 \mathrm{D}$-printing process was found in the first round of experiments. In this first round, bilayer polymer structures with different layer heights were printed and tested. Numerical simulation results with an initial stress of $270 \mathrm{kPa}$ showed an optimal agreement with the experimental results, and therefore this stress value was chosen as the calibrated prestress. After calibration of the prestress, the modeling approach was validated using the same experimental results. It can be concluded that the numerical model performs well for uniform bilayered structures with layer sizes of 0.5 and $1 \mathrm{~mm}$. From the experiments with topology optimized designs can be learned that gravitational effects cannot be neglected, and that other environmental influences may also affect the bending behaviour. Overall, the printed optimized designs show a qualitative agreement with the simulated results.

To further improve on the presented approach to use topology optimization for 4D-printed structures, many opportunities for future work exist. Some of those opportunities involve changes to the modeling assumptions that were made. It could be investigated if a hyperelastic material model obtains results that resemble the experimental results better than a linear elastic material model. Also, the role played by anisotropy in the printed materials can be looked into. Factors in the testing environment, such as viscosity of the water, gravity and heat transfer are expected to also play a role. These factors were all neglected in the current work. Doing more experiments with a larger number of samples would help in getting a greater insight in the accuracy of the measurements, the factors influencing the prestress, and the performance of the modeling approach. The modeling and topology optimization approach can be taken to a next level by aiming for more complex target displacements. To further increase the design freedom of the 
optimizer, a material optimization could be used instead of using fixed material domains as a starting point for the topology optimization. By doing this, structures of increased complexity can be generated, such that in the end potential applications such as self-(dis)assembly of products may be realized.

\section{REFERENCES}

1 Ngo, T. D., Kashani, A., Imbalzano, G., Nguyen, K. T., and Hui, D. (2018). Additive manufacturing (3D printing): A review of materials, methods, applications and challenges. Composites Part B: Engineering, 143(December 2017):172-196.

2 Tibbits, S. (2014). 4D printing: Multi-material shape change. Architectural Design, 84(1):116-121.

3 Momeni, F., M.Mehdi Hassani.N, S., Liu, X., and Ni, J. (2017). A review of 4D printing. Materials and Design, 122:42-79.

4 Bendsoe, M. and Sigmund, O. (2003). Topology Optimization - Theory, Methods and Applications. Springer-Verlag Berlin Heidelberg.

5 Maute, K., Tkachuk, A., Wu, J., Qi, H. J., Ding, Z., and Dunn, M. L. (2015). Level Set Topology Optimization of Printed Active Composites. Journal of Mechanical Design, Transactions of the ASME, 137(11).

6 Geiss, M. J. and Maute, K. (2018). Topology optimization of active structures using a higher-order level-set-XFEM-density approach. 2018 Multidisciplinary Analysis and Optimization Conference, pages 1-13.

7 Sossou, G., Demoly, F., Belkebir, H., Qi, H. J., Gomes, S., and Montavon, G. (2019). Design for 4D printing: Modeling and computation of smart materials distributions. Materials and Design, 181:108074.

8 Geiss, M. J., Boddeti, N., Weeger, O., Maute, K., and Dunn, M. L. (2019). Combined Level-Set-XFEM-Density Topology Optimization of Four-Dimensional Printed Structures Undergoing Large Deformation. Journal of Mechanical Design, Transactions of the ASME, 141(5):1-14.

9 Bodaghi, M., Damanpack, A. R., and Liao, W. H. (2016). Selfexpanding/shrinking structures by 4D printing. Smart Materials and Structures, $25(10)$.

${ }^{10}$ Akbari, S. and Sakhaei, A. H. and Kowsari, K. and Yang, B. and Serjouei, A. and Yuanfang, Z. and Ge, Q. (2018). Enhanced multimaterial 4D printing with active hinges. Smart Materials and Structures, 27 
${ }^{11}$ Chen, T., Bilal, O. R., Lang, R., Daraio, C., and Shea, K. (2019). Autonomous Deployment of a Solar Panel Using Elastic Origami and Distributed ShapeMemory-Polymer Actuators. Physical Review Applied, 11(6).

${ }^{12}$ Inverardi, N., Pandini, S., Bignotti, F., Scalet, G., Marconi, S., and Auricchio, F. (2020). Sequential Motion of 4D Printed Photopolymers with Broad Glass Transition. Macromolecular Materials and Engineering, 305(1):1-11.

${ }^{13}$ Ding, Z., Yuan, C., Peng, X., Wang, T., Qi, H. J., and Dunn, M. L. (2017). Direct 4D printing via active composite materials. Science Advances, 3(4).

${ }^{14}$ Mahieux, C. A. and Reifsnider, K. L. (2001). Property modeling across transition temperatures in polymers: A robust stiffness - Temperature model. Polymer, 42(7):3281-3291.

${ }^{15}$ Selva Priya, M., Kakur, N., Rengaswamy, J., Ramachandran, V. (2019). A comparative study between in-house 3D printed and injection molded ABS and PLA polymers for low-frequency applications. Materials Research Express, 6(8).

${ }^{16}$ Xiao, R., Choi, J., Lakhera, N., Yakacki, C., Frick, C., Nguyen, T. (2013). Modeling the glass transition of amorphous networks for shape-memory behavior. Journal of the Mechanics and Physics of Solids, 61(7):1612-1635.

${ }^{17}$ Bendsoe, M. P. (1989). Optimal Shape Design as a Material Distribution Problem. Structural Optimization, 1:193-202.

${ }^{18}$ Lazarov, Boyan Stefanov and Ole Sigmund (2011). Filters in topology optimization based on Helmholtz-type differential equations. International Journal for Numerical Methods in Engineering, 86(6):765-781.

${ }^{19}$ Wang, Y., Luo, Z., Kang, Z., and Zhang, N. (2015). A multi-material level setbased topology and shape optimization method. Computer Methods in Applied Mechanics and Engineering, 283:1570-1586. 